\title{
Optical System Design of the National Ignition Facility
}

\author{
R. E. English \\ C. W. Laumann \\ J. L. Miller \\ L. G. Seppala \\ This paper was prepared for submittal to the \\ Optical Society of America 1998 Summer Topical Meetings \\ Kailuia-Kona, HI \\ June 8-12, 1998
}

June 26, 1998

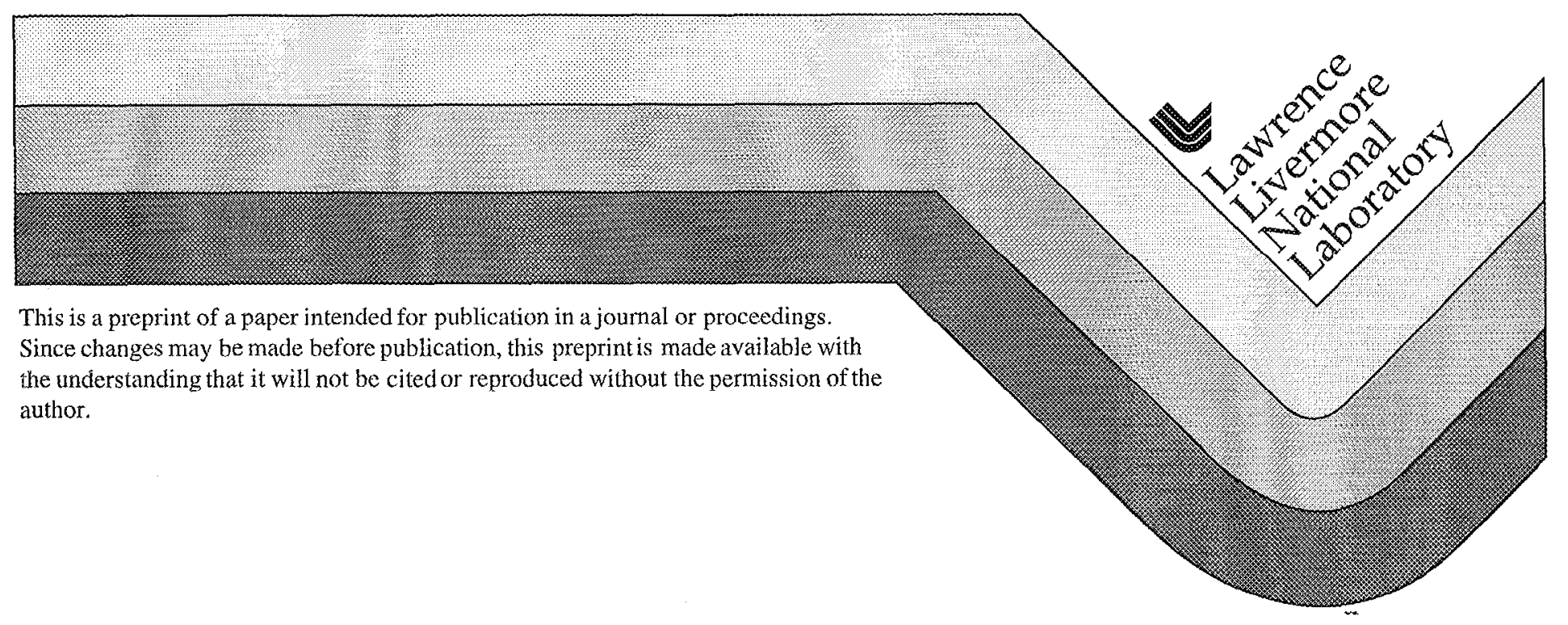




\section{DISCLAIMER}

This document was prepared as an account of work sponsored by an agency of the United States Government. Neither the United States Government nor the University of California nor any of their employees, makes any warranty, express or implied, or assumes any legal liability or responsibility for the accuracy, completeness, or usefulness of any information, apparatus, product, or process disclosed, or represents that its use would not infringe privately owned rights. Reference herein to any specific commercial product, process, or service by trade name, trademark, manufacturer, or otherwise, does not necessarily constitute or imply its endorsement, recommendation, or favoring by the United States Government or the University of California. The views and opinions of authors expressed herein do not necessarily state or reflect those of the United States Government or the University of California, and shall not be used for advertising or product endorsement purposes. 


\title{
Optical system design of the National Ignition Facility
}

\author{
R. Edward English Jr., Curt W. Laumann, John L. Miller, Lynn G. Seppala \\ Lawrence Livermore National Laboratory \\ PO Box 808, L-527 \\ Livermore, CA 94551 \\ (925) 422-3602, (925) 424-6085 fax, english2@llnl.gov
}

\begin{abstract}
The National Ignition Facility (NIF) is a laser fusion facility being constructed at Lawrence Livermore National Laboratory (LLNL). The neodymium-doped phosphate glass pulsed laser system will produce over $3.5 \mathrm{MJ}$ of laser energy at a fundamental lasing wavelength of $1.053 \mu \mathrm{m}$ $(1 \omega)$. The final optics assembly contains a pair of crystals $(\mathrm{KDP} / \mathrm{KD} * \mathrm{P})$ and a focusing lens to convert the light by sum-frequency-mixing to $3 \omega(\lambda=0.35 \mu \mathrm{m})$ and focus $1.8 \mathrm{MJ}$ onto the target.

The NIF optical system is large and complex. To give some perspective the NIF building is roughly 200 meters long x 85 meters wide. There are approximately 7500 optical components in the large aperture laser system - lenses, mirrors, polarizers, laser slabs, crystals, and windows cach with a clear aperture greater than $40 \mathrm{~cm}$ square. The front-end of the laser system contains more than 8000 srnaller $(5-15 \mathrm{~cm})$ precision laser components.

In this paper we will describe the optical system configuration, layout, and general design considerations. We will explain the path of the pulse through the various subsystems. Some of the top-level optical system and sub-system design requirements will be presented.
\end{abstract}

\section{Introduction}

The NIF system comprises 192 independent laser beams. They are stacked four high, and two columns are grouped and can be operated as a "bundle" ( $4 \times 2$ laser beams); there are 24 bundles. Because of this arrangement, the laser beam footprint is square and adjacent beams are relatively close together to optimize the extraction of energy from the amplifier.

The optical system design, which is nearing completion, has proceeded by considering the NIF to comprise six main subsystems: optical pulse generation, injection, main laser, switchyard and target area, final optics assembly, and beam control (see Fig. 1). A summary of large aperture optical components is presented in Table 1 near the end of this paper.

\section{System architecture and path of a photon}

\section{Optical pulse generation system}

The optical pulse generation (OPG) system produces the injected pulse for the main laser system. The output from a single, fiber laser oscillator is amplified, split into 48 fibers, temporally shaped with integrated optics modulators, and distributed via fibers to the preamplifier modules (PAM). The energy at this point is a few nanojoules. 
By means of a regenerative amplifier and a four-pass rod amplifier, the pulse is amplified first to an energy of about $10 \mathrm{~mJ}$ and then to an energy of 10-20J. Before the rod amplifier section, the beam aperture is defined by a serrated aperture, $22.5 \mathrm{~mm} \times 22.5 \mathrm{~mm}$. This plane is subsequently relayed to key points (e.g., rod amplifier, cavity end mirrors) within the optical system by afocal telescopes that also perform spatial filtering. This is a fundamental requirement of the optical system design.

The detailed configuration of the optical layout of the four-pass rod amplifier system is shown in Fig. 2. The raytrace model has been used to define the optical configuration, convey information to the mechanical design team regarding the detailed position of optical components, analyze clear aperture requirements under expected installation and alignment tolerances, perform lens design optimization, generate lens prescriptions, and perform tolerance analyses.

There are 48 preamplifier modules; the multi-joule output from each must be split into four beams before injection into the main laser system. This splitting is accomplished by a cascade of half-waveplates and polarizers that first generate two, then four beams. These four beams contain up to $3 \mathrm{~J}$ of energy and are independently controlled/aligned through the rest of the system. A multi-element afocal telescope for beam transport follows this 1:4 split. The telescope is nominally unit magnification, but it has been designed to so that slight adjustments to beam size $(+5 \% /-2 \%)$ and collimation of the beam injected into the main cavity can be made by changing the spacing of lenses in the telescope.

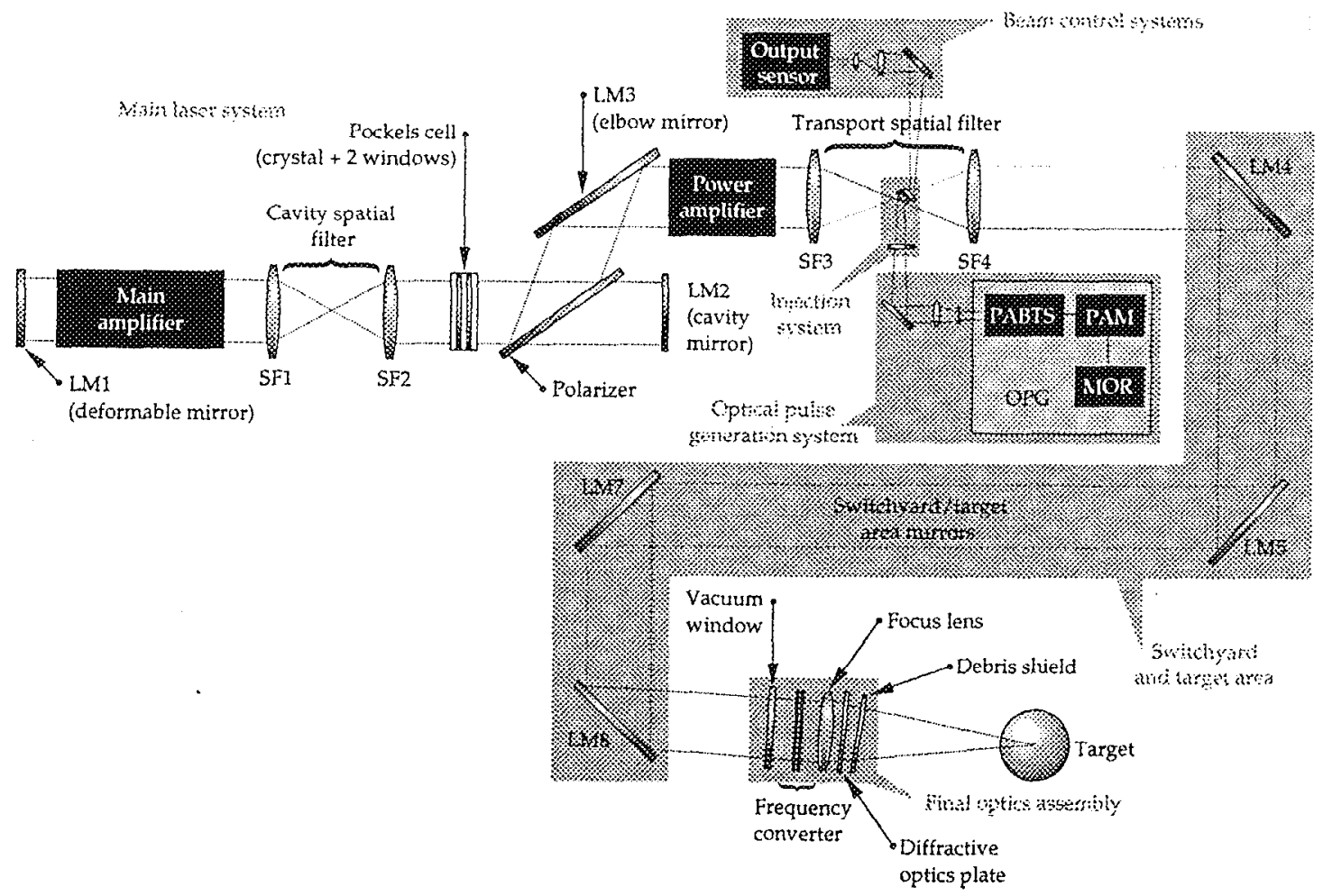

Fig. 1. This schematic illustrates the 6 main optical systems of the National Ignition Facility: optical pulse generation, injection, main laser, switchyard and target area, final optics, and beam control. 


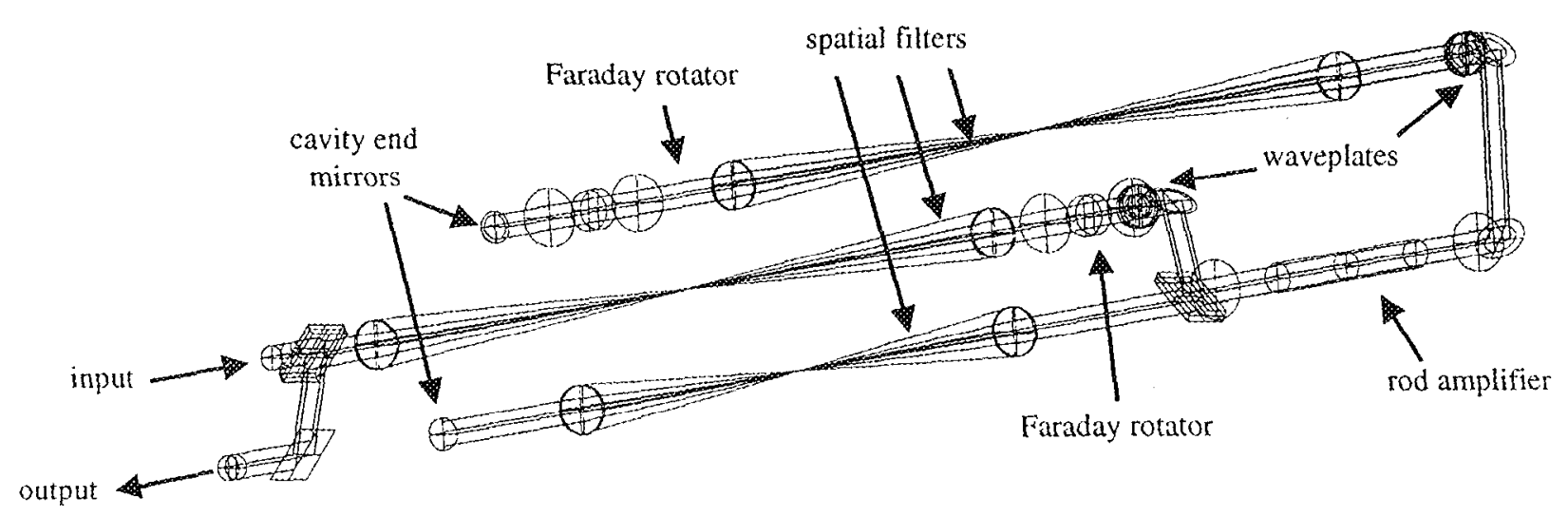

Fig. 2. In the four-pass rod amplifier system in the preamplifier module (PAM), light enters the cavity through a polarizer (left-side of figure). A telescope relays the beam aperture to the center of the 5-cm diameter rod amplifier (first pass). Note that the pulse has also passed through a Faraday rotator and half-waveplate. A second telescope relays the aperture onto the first cavity end mirror and then back into the rod amplifier (second pass). (There is a second Faraday rotator in this part of the cavity for suppression of parasitics and decreased sensitivity to birefringence effects.) Two passes through the quarter-waveplate in the upper leg of the cavity permits the pulse to transmit through a polarizer in the lower leg of the cavity. A third telescope and second cavity end mirror relay the beam back through the amplifier (third pass). One more trip through the upper part of the cavity sends the pulse through the amplifier for its fourth pass. Now the light is reflected off the polarizer, transmits through the Faraday rotator and telescope, and exits the module.

\section{Injection system}

The injection system links the optical pulse generation system with the main laser system. The injection system is a telephoto lens pair that matches the f/no of the transport spatial filter lens, thereby producing the desired beam size in the main system ( $f / n o=57$, beam size $=372 \mathrm{~mm} \mathrm{x}$ $372 \mathrm{~mm}$ ). This lens pair and a succeeding mirror are positioned relatively close to the pinhole plane of the transport spatial filter. The function of this system will become more clear after a discussion of the main laser system.

\section{Main laser system}

The NIF main laser cavity is formed by two mirrors and a 1:1 afocal telescope (cavity spatial filter) whose length is $23.5 \mathrm{~m}$, one-half the cavity length of $47 \mathrm{~m}$. (Another way to think of the optical system is that the end mirrors are imaged telecentrically onto each other.) Generally, on high peak-power laser systems, a spatial filter comprises two lenses in an afocal configuration. 
The lens are at either end of a vacuum vessel or tube and function as a vacuum barrier. Pinholes at the focal plane "scrape off" unwanted, high-angle diffracted and scattered light to prevent nonlinear growth of scattered waves that would produce intensity modulation and damage optical components. In the NIF main laser system, these lenses are fused silica, equi-convex, aspheres with a clear aperture slightly larger than $40 \mathrm{~cm}$ square. ${ }^{1}$

The cavity or main amplifier is four-passed; the booster or power amplifier is two-passed. Neodymium-doped, phosphate laser glass is the material for the amplifier slabs; for each beam, there are 11 slabs in the main amplifier and 5 slabs in the power amplifier. They are oriented at Brewster's angle to minimize loss (the slabs are uncoated) and are pumped with flashlamps that are long enough to pump the four-slab high amplifier. (Laser beams are stacked 4-high; two columns are grouped together in a $4 \times 2$ bundle.) Copper-doped phosphate glass is epoxy-bonded onto the edges of each slab to absorb ASE that propagates transverse to the laser beam.

One end mirror is a 39-actuator deformable mirror; its primary purpose is correction of pumpinduced, cladding-absorbed ASE that causes thermal aberrations in the phosphate laser glass amplifier slabs. Additionally, the mirror corrects passive optical fabrication and installation errors.

The main laser system also contains a transport spatial filter. This $60 \mathrm{~m}$ long transport spatial filter telescope (two lenses, each with $30 \mathrm{~m}$ focal length) has several functions that will be explained as we continue to trace the path of a photon through the system.

Up to $3 \mathrm{~J}$ of energy can be injected into each beamline. The preamplifier module and beam transport systems (e.g., 1:4 split) are located beneath the transport spatial filter. The beams enter the vacuum system through windows in the bottom of the vacuum vessel, and the injection system directs light toward the main cavity by focusing it through a pinhole located $30 \mathrm{~m}$ from the transport spatial filter lens. The light is collimated by the spatial filter lens and propagates through the power amplifier. The beam defining aperture is relayed toward the main cavity, too.

The beam changes elevation after reflection from a mirror and polarizer, each at Brewster's angle for BK-7, which is the substrate for mirrors and polarizers in the system. The manner in which the 4-high beams propagate into the cavity is shown in Fig. 3. Detailed raytrace models have been developed and used to ensure that the components are properly positioned in the mechanical mounts and structures for optimal use of the available clear aperture of the elements. The polarization state is horizontal for high transmission through the amplifier slabs. The Pockels cell is initially off (i.e., does not rotate the polarization state) as the pulse enters the main cavity.

The beam aperture is relayed to the cavity end mirror by the cavity spatial filter. (This linkage with the optical pulse generation system beam transport and injection systems is controlled by optical interface specifications and an integrated raytrace model now in final study.) After the beam has made two passes through the main amplifier (beam energy approximately 500J), it passes once more through the spatial filter. By now, the Pockels cell has switched on so that it rotates the polarization state by 90 degrees. Thus, the pulse transmits through the polarizer with high transmission, reflects off the second cavity mirror, and transmits through the polarizer a second time. The Pockels cell is still on for the third pass; this rotates the polarization state back 
to horizontal for the third and fourth passes through the main amplifier. The beam energy is now about $11 \mathrm{~kJ}$. When the pulse returns to the Pockels cell a fourth time, it has switched off, and the beam reflects off the polarizer and out of the cavity. A second (and final) pass through the power amplifier brings the beam energy up to about $18 \mathrm{~kJ}$.

The transport spatial filter now performs its next tasks: projection of the beam-defining aperture toward the final optics assembly and final beam clean-up. This beam clean-up is especially important here because of the high peak irradiance. Nonlinear (i.e., irradiance-dependent) index of refraction phase gradients build-up substantially in the last two slabs and input lens to the transport spatial filter lens. The slabs are $41 \mathrm{~mm}$ thick at Brewster's angle and the lens is $46 \mathrm{~mm}$ thick; the peak irradiance approaches $5 \mathrm{GW} / \mathrm{cm}^{2}$. The delta-B (a measure of this nonlinear index growth) is approximately $1.6-1.8$ radians before the spatial filter. The final pinholes have an angular extent of $+/-100 \mu \mathrm{rad}$ to filter out unwanted high spatial frequencies and reduce the modulation that would arise from these phase gradients.

A brief description is needed to understand how the pinholes are amanged in the spatial filters. The injection pinhole is offset $17.5 \mathrm{~mm}$ laterally from the optical axis of the input transport spatial filter lens. Thus, the beam goes through this lens off-axis. The optical axis of the cavity spatial filter is offset vertically from the transport spatial filter's optical axis. The first cavity end mirror is perpendicular to the cavity spatial filter axis, but the second cavity end mirror is tilted slightly down. The alignment is such that the four passes through the cavity are through four pinholes separated by about $13.7 \mathrm{~mm}$; the centroid of these four pinholes is on the cavity spatial

ELBOW MIRROR (LM3)

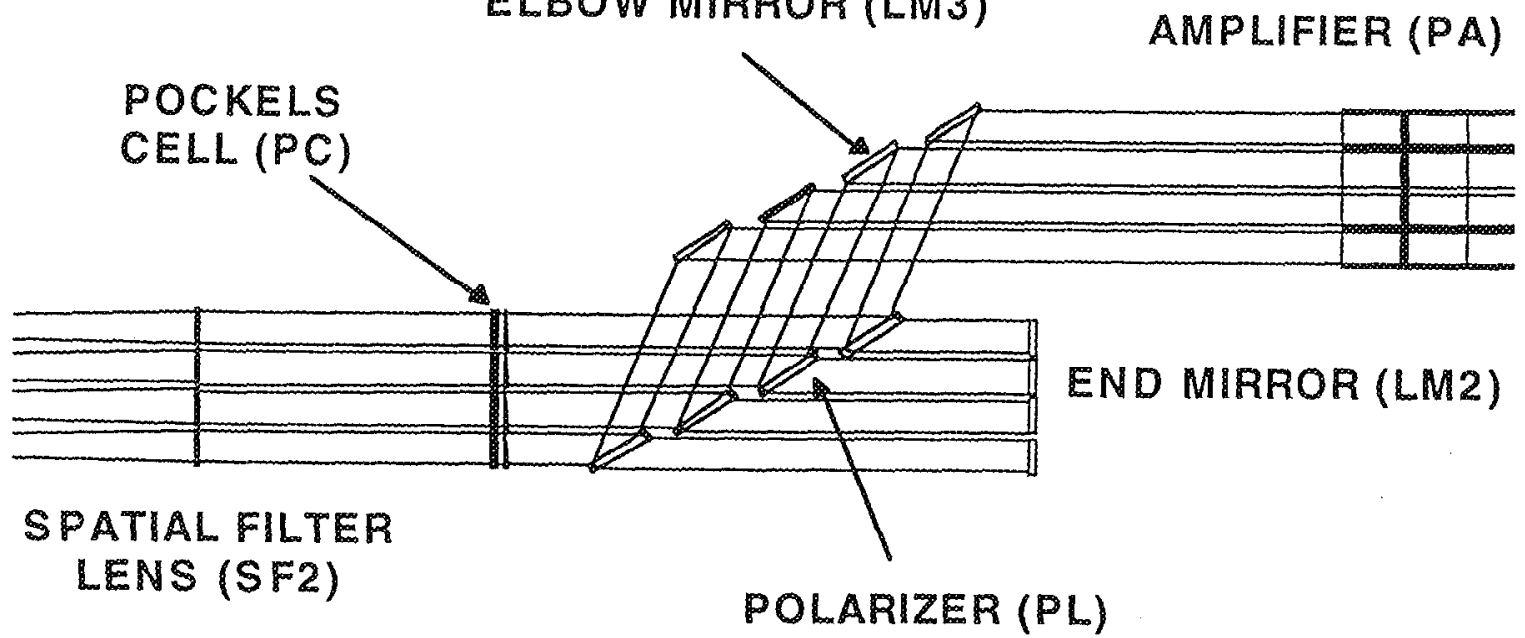

Fig. 3. The periscope region (shown here in elevation) is where the beam is switched in and out of the main laser cavity by the action of the Pockels cell and polarizer. Two laser slabs are shown in the power amplifier, rotated about a vertical axis. The polarization state is horizontal for high transmission through the slabs and reflection from the polarizer into the cavity. The Pockels cell is initially off (first pass), then on (second and third pass), then off again (fourth pass) so that the pulse makes four passes through the main amplifier (two round trips in the cavity). 
filter axis. The beam exiting from the main cavity goes through its final pinhole in the transport spatial filter offset $17.5 \mathrm{~mm}$ in the other direction from the injected beam (separation of these two pinholes is $35 \mathrm{~mm}$ ). The arrangement of the pinholes is shown in Fig. 4.

A complete lens model was developed for the laser beam in the main laser system, which is $123 \mathrm{~m}$ long. A plot from the lens design code is not illustrative because the system is so long compared to its transverse dimension. The main version of this model, developed using a commerciallyavailable optical design tool, is a sequential lens file with over 200 surfaces that account for all of the tilts and decenters within the system. The detailed model has been an instrumental tool for opto-mechanical configuration control, alignment and control system modeling, clear aperture checking, and lens design tolerancing.

\section{Switchyard and target area mirror system}

As has been mentioned, the beams are grouped in bundles of 8 beams; bundles can be operated and fired independently. In addition, 6 bundles are clustered together so that there are two such clusters in each of two laser bays. The arrangement of beams is in a rectilincar grid for tight packing, minimum building footprint, and amplifier efficiency.

To meet the requirements of symmetric target illumination, however, the beams must be arranged in a polar geometry about the $10 \mathrm{~m}$ diameter target chamber. This is accomplished in the switchyard and target area by a system of 832 fold mirrors. First, bundles are "scraped off" into quads, which are $2 \times 2$ groupings. These quads are then routed to the target chamber. Target physics symmetry requires illumination from two cones on the top and two cones on the bottom (one cone at about 20 degrees and one at about 50 degrees, measured from the pole of the chamber). There are eight quads in the inner cone and 16 quads in the outer cone.

\section{CSF}

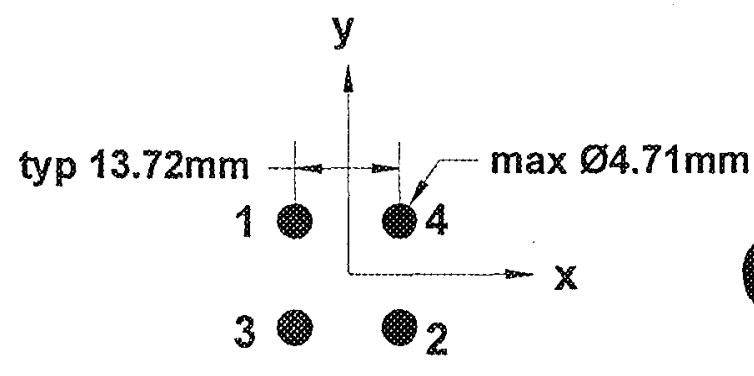

TSF

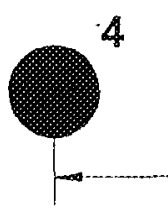

y 
Each beam path has at least four mirrors; because of geometry and space constraints, some have five. The reflections are nominally s- or p-polarization. There are variations in path length (beamto-beam) from the main laser system to the final optics system. In the final design, this path length ranges from $62 \mathrm{~m}$ to $74 \mathrm{~m}$. All of the mirrors are remotely actuated in two orthogonal axes; however, only four degrees of freedom (mirror tilts) are used to control pointing to the target and centering at the final optics assembly. The apertures of the mirror must accommodate the laser beam footprint, alignment and installation allowances, and transport of a back-reflected diagnostic signal from the final optics. In addition, the apertures must allow decentering of the laser beam on mirrors needed to meet the requirement to point beams within $+1.30 \mathrm{~mm}$ of the center of the target chamber.

In the main laser system, the optical train is the same for each of the 192 beams (with the exception of slight differences in the periscope region). In the switchyard and target area mirtor system, however, optical models had to be constructed for each of the 192 beam lines; see Fig. 5 . (There are some symmetries that simplify the required analyses somewhat.) The macro languages available in current lens design codes greatly assisted in this task. Nevertheless, the nature of this system (e.g., the 3-D geometry, beam-to-beam space constraints, need to minimize mirror sizes, development of alignment control algorithm) made the design of this flat mirror system a relatively complex optical design task .

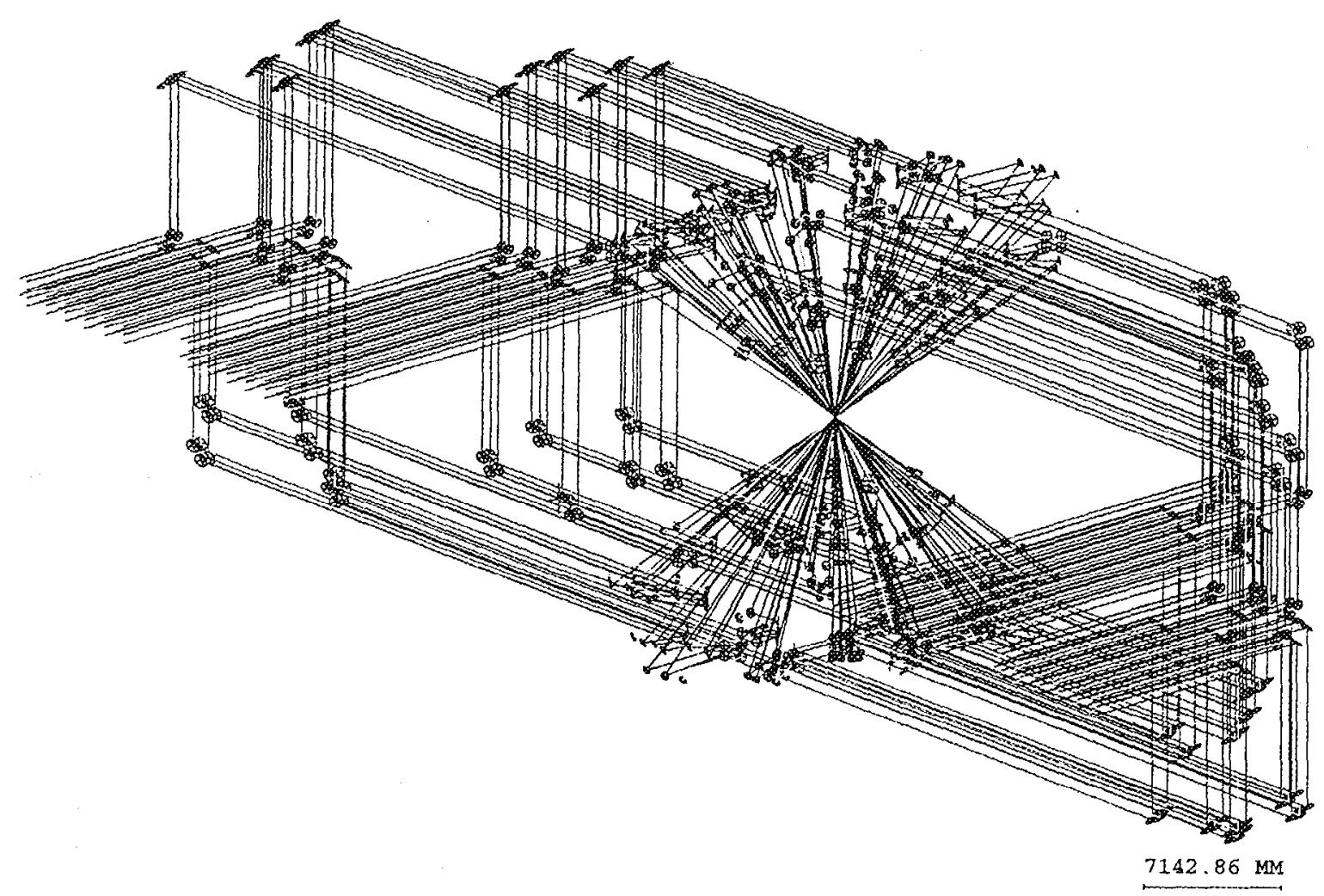

Fig. 5. The switchyard and target area mirror system contains 832 fold mirrors that map the rectilinear geometry in the laser bay to the spherically symmetric geometry required for illuminating the target. Optical models were constructed for each beam. 


\section{Final optics assembly}

The primary functions of the final optics assembly are to convert the laser light to $3 \omega$ and focus it on target. Four beams come into one assembly that contains four separate sets of optics. Each beam passes through a vacuum window (the target chamber is at vacuum), a pair of frequency conversion crystals (KDP/KD*P), a focus lens (focal length $7.7 \mathrm{~m}$ ), two diffractive optics plates, and a debris shield; see Fig. 6.

The basic design of the focus lens is completely determined by overall system requirements and architecture. The focal length was established as a compromise between a) dividing the desired focal spot (unsmoothed) by the expected laser beam quality and b) having sufficient mechanical clearance between assemblies mounted to a $10 \mathrm{~m}$ diameter sphere.

The lens shape is dictated by the alignment scheme for frequency conversion. The doubler (KDP) and tripler $\left(\mathrm{KD}^{*} \mathrm{P}\right)$ in this Type I/Type II frequency conversion scheme are sensitive in orthogonal axes with regards to their phase-matching angle. Thus, a single cell can be tilted to orient both crystals properly with respect to the incoming $1 \omega$ beam. The crystals are not flat enough, however, to provide a high fidelity alignment reference signal themselves (reflected from their surface) to the aligniment sensor. Hence, the focus lens is plano-convex lens and is mounted in the cell with its flat surface in collimated space and all the bending occurring at the convex surface. (This is, of course, "backwards" from conventional practice.) It means that the aspheric, convex surface has a steeper departure $(\sim 8 \mu \mathrm{m})$ and narrower field-of-view $(\sim+1-200 \mu \mathrm{rad})$ than would otherwise be the case; however, the cell is always tilted so that the laser beam is entering at the proper angle, and the narrow field-of-view is not a factor.

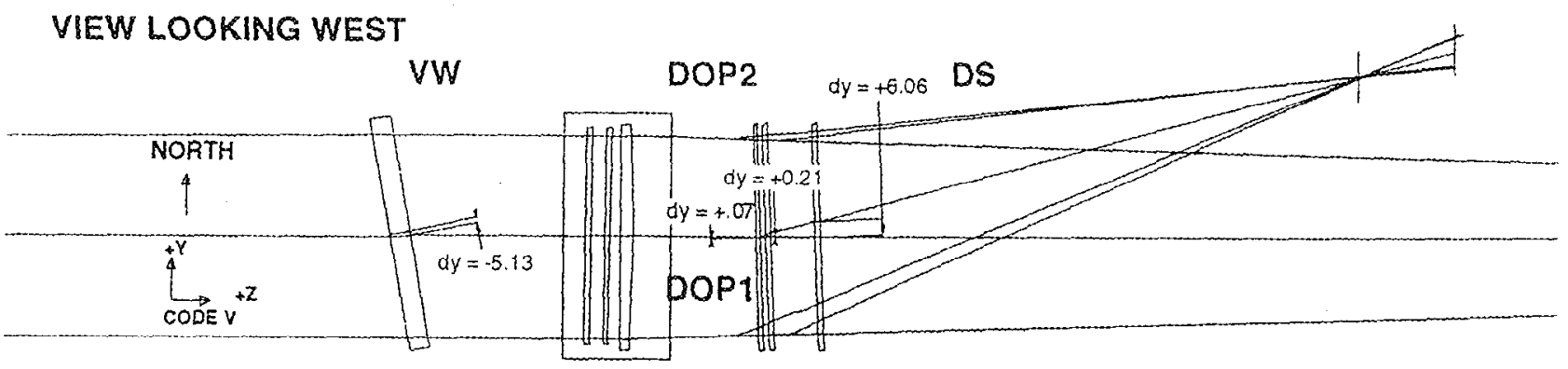

VIEW LOOKING SOUTH

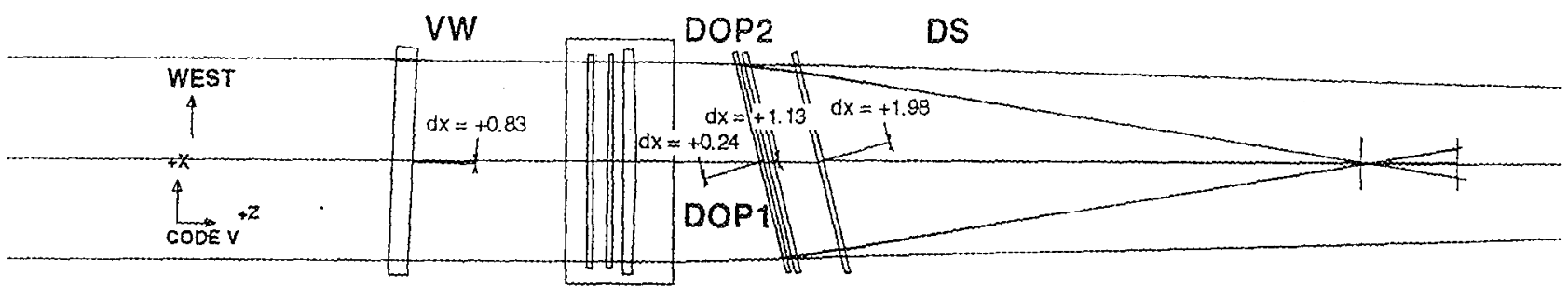

Fig. 6. The final optics assembly contains a vacuum window (VW), two frequency conversion crystals and a focus lens in a single cell, two diffractive optics plates (DOP1 and DOP2) and a debris shield (DS). The first-order diffracted beam sample is shown coming to focus to a point 14 degrees off-axis and a back focal length of $1.2 \mathrm{~m}$. The debris shield protects the final optics from target debris and contamination. 
The final optics assembly includes diffractive optical elements; three diffractive functions are planned to be incorporated onto two flat plates after the lens: beam sampling ( $3 \omega$ energy), beam smoothing ( $3 \omega$ on target), and unconverted light management (moving $1 \omega / 2 \omega$ away from the target). Beam sampling is achieved by a low-efficiency grating with off-axis focusing power to focus $0.2 \%$ of the $3 \omega$ energy onto a calorimeter for measurement of energy on target. A kinoform phase plate produces the desircd irradiance profile on the target, namely an elliptically shaped, top-hat far-field pattern. Even though the frequency conversion efficiency is high for NIF ( $60 \%$ energy and $85 \%$ at the peak of the pulse), a substantial amount of unconverted light remains (especially $1 \omega$ ). This light will focus past the target, but, if undiverted, the irradiance is sufficient to interfere with the target experiment. A color separation grating will be employed. This is a two-step, linear grating with period approximately $0.5 \mathrm{~mm}$ that diverts the $1 \omega / 2 \omega$ light at least $10 \mathrm{~mm}$ away from the target. The two steps are $2 \pi$ and $4 \pi$ phase steps for $3 \omega$. In theory, such a grating diffracts no $l \omega$ or $2 \omega$ into the zeroeth-order. In practice, efficiencies of $<1 \%$ have been demonstrated; this is sufficient for adequate system performance.

The relative positions of the optical components within the final optics assembly were determined by careful analysis of ghost reflections. This analysis is complicated by the 14 surfaces involved, the relatively close spacing of components, the mechanical restrictions in spacing the components, the high fluence (at both $1 \omega$ and $3 \omega$ ), the focusing power, dispersion, and multi-order diffraction from the beam sampling grating, the range of travel required for focus lens adjustment $(+/-50 \mathrm{~mm})$, and tolerances on manufacturing. The vacuum window before the lens and diffractive optics plates and debris shield after the lens are tilted substantially so that their ghost reflections "walk" out of the beam footprint. Literally, over a million ghosts were analyzed by state-of-the-art, non-sequential raytrace codes that tracked energy splitting up to fourth-order reflections.

\section{Beam control systems}

The sixth optical "subsystem" is really a number of optical systems that are part of the beam control systems. The primary sensor package is the output sensor; the optical configuration is shown in Fig. 7 on the last page. (The other sensors will not be discussed here.) It is located beneath the center of the transport spatial filter, and it receives optical signals from three locations.

A diagnostic beamsplitter (not shown in Fig. 1) is part of the main beam and immediately follows the output lens of the transport spatial filter. The full aperture reflection from this optical component is used for alignment, diagnostics, and wavefront control of the main laser system. The full aperture reflection from the focus lens is used for alignment and diagnostics (previously mentioned). The output spatial filter lens is the first part of the beam reducing telescope for these diagnostic samples. These two beam samples are routed via relay telescopes to the output sensor. In addition, a third set of relay telescopes provides viewing of the pinhole plane for alignment. 


\section{References}

1. R. Korniski et al., "Spatial filter lens design for the main laser of the National Ignition Facility," 1998 International Optical Design Conference, paper LFB2.

*This work was performed under the auspices of the U.S. Department of Energy by Lawrence Livermore National Laboratory under Contract No. W-7405-Eng-48.

Table 1. Large aperture optical components for NIF

\begin{tabular}{|c|c|c|c|}
\hline Component & Quantity & Material & Size $(\mathrm{mm})$ \\
\hline Amplifier slabs & 3072 & phosphate glass & $808 \times 458 \times 41$ \\
\hline Spatial filter lenses & 768 & fused silica & $438 \times 434 \times 46$ \\
\hline Focus lenses & 192 & fused silica & $430 \times 430 \times 25$ \\
\hline Cavity mirrors & 384 & BK-7 & $412 \times 412 \times 80$ \\
\hline Elbow mirrors & 192 & $\mathrm{BK}-7$ & $417 \times 740 \times 80$ \\
\hline Transport mirrors & 832 & $\mathrm{BK}-7$ & $\begin{array}{l}\text { various sizes } \\
\text { minimum dim. } 440 \\
\text { maximum dim. } 690 \\
\text { all } 80 \text { thick }\end{array}$ \\
\hline Polarizers & 192 & $\mathrm{BK}-7$ & $417 \times 807 \times 90$ \\
\hline Crystals & 576 & $\mathrm{KDP} / \mathrm{KD} * \mathrm{P}$ & $410 \times 410 \times 10^{*}$ \\
\hline Switch windows & 384 & fused silica & $430 \times 430 \times 35$ \\
\hline Target chamber windows & 192 & fused silica & $450 \times 440 \times 43$ \\
\hline Diffractive optics plates & 384 & fused silica & $430 \times 430 \times 10$ \\
\hline Debris shields & 192 & fused silica & $430 \times 430 \times 10$ \\
\hline Diagnostic beamsplitter & 192 & fused silica & $438 \times 434 \times 10$ \\
\hline
\end{tabular}




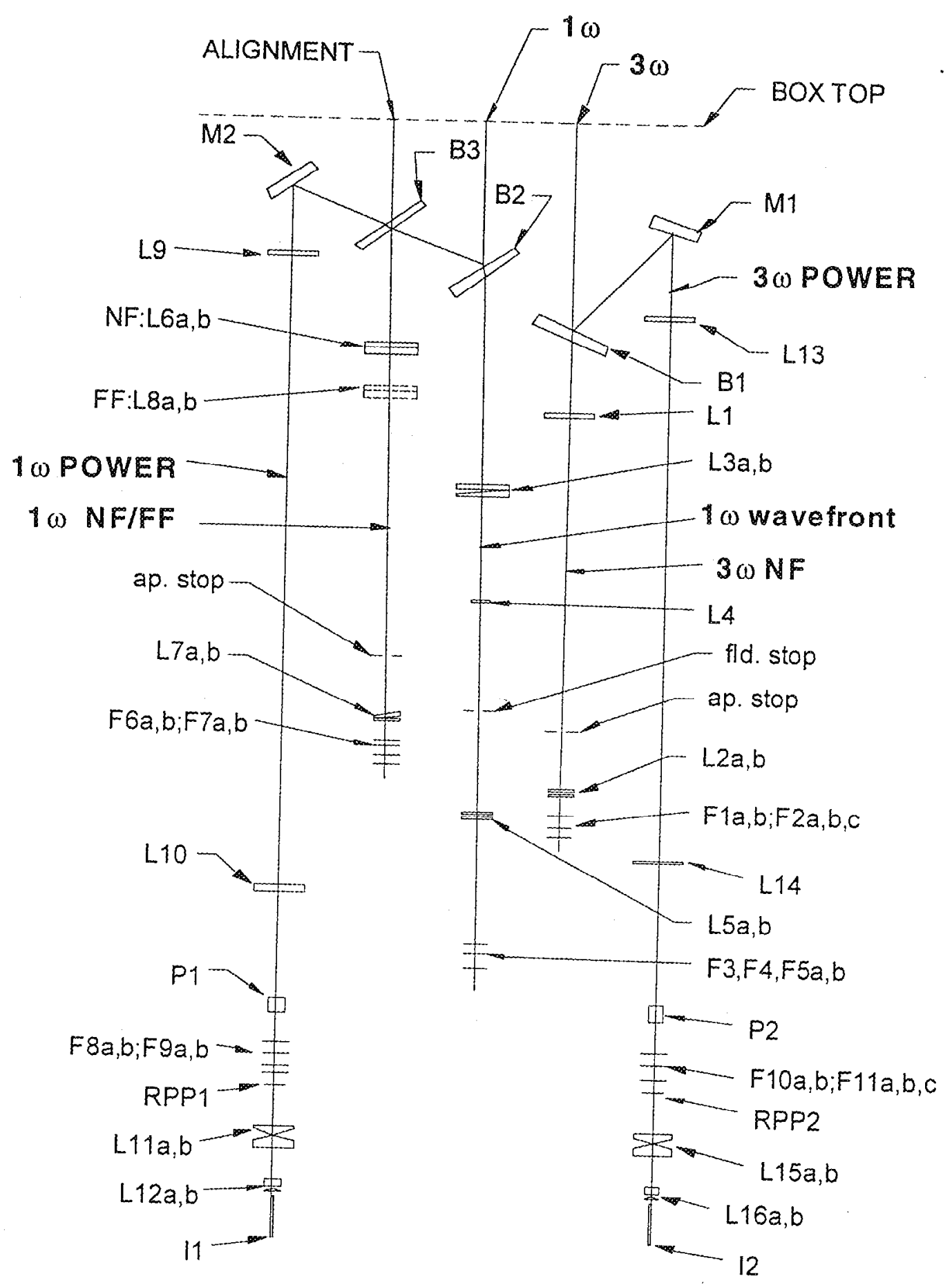

Fig. 7. The output sensor performs a variety of alignment and diagnostic functions. This optical layout (gut ray trace shown) contains optical paths for alignment (beam centering within the aperture and pointing to pinholes), far-field for $(1 \omega / 3 \omega)$, near-field $(1 \omega / 3 \omega)$, wavefront $(1 \omega)$, energy $(1 \omega)$, and pulse shape $(1 \omega / 3 \omega)$. There are $\sim 50$ optical components in each output sensor package. A single package is multiplexed between two beams (total of 96 packages). 